\title{
Identification of Molecular Markers Linked to the Trait of Natural Astringency Loss of Japanese Persimmon (Diospyros kaki) Fruit
}

\author{
Shinya Kanzaki, ${ }^{1}$ Keizo Yonemori, ${ }^{2}$ and Akira Sugiura \\ Laboratory of Pomology, Graduate School of Agriculture, Kyoto University, Sakyo-ku, \\ Kyoto 606-8502, Japan

\begin{abstract}
Akihiko Sato and Masahiko Yamada
Persimmon and Grape Research Center, National Institute of Fruit Tree Science, Akitsu, Hiroshima 7292494, Japan
\end{abstract}

AdDitional INDEX wORDS. AFLP, bulked segregant analysis, astringency, RFLP marker

\begin{abstract}
Aвstract. Japanese persimmon (Diospyros kaki Thunb.) cultivars are classified into four types depending upon the nature of astringency loss of the fruit. Among them, the pollination-constant and nonastringent (PCNA) type is the most desirable for fresh fruit consumption due to the trait of stable loss of astringency on the tree with fruit development. Lack of tannin accumulation is the main cause of natural astringency loss in PCNA-type fruit, and is qualitatively inherited. The PCNA trait is recessive to the non-PCNA trait. In this study, we investigated amplified fragment length polymorphism (AFLP) markers for the trait of natural astringency loss of PCNA-type fruit using bulked segregant analysis (BSA) for efficient selection of PCNA type plants in a breeding population. A total of 128 primer combinations were tested and one AFLP marker was found to be linked to the dominant allele controlling the trait for astringency. This marker, EACC/ MCTA-400, was absent in all of the PCNA-type plants tested, whereas it was present in about half of the non-PCNA-type plants tested. However, RFLP analysis using this marker enabled the detection of the other dominant allele, and all PCNA-type plants could be distinguished from the non-PCNA-type plants. Application of this marker system will be useful for the selection of PCNA-type plants in persimmon breeding.
\end{abstract}

Japanese persimmon (Diospyros kaki) cultivars are classified into four types depending on classification of astringency, presence of seeds, and flesh color (Kajiura, 1946; Kikuchi, 1948). These four types are 1) pollination-constant and nonastringent (PCNA), 2) pollination-variant and nonastringent (PVNA), 3) pollination-variant and astringent (PVA), and 4) pollinationconstant and astringent (PCA). Both the PVA and PCA types have astringent fruit at maturity and are edible only after the astringency has been removed by artificial treatment with ethanol, $\mathrm{CO}_{2}$ or drying. Although some cultivars of these types may produce high-quality fruit, the need of costly facilities for astringency removal makes it economically difficult to expand the cultivation area of these types. Although the other two (PCNA and PVNA) types have fruit losing astringency naturally with fruit growth and are edible at maturity without any artificial treatment, the loss of astringency in PVNA-type fruit is dependent on the existence of seeds in the fruit. If pollination is insufficient and the fruit contains few or no seeds, PVNA-type fruit does not lose astringency on the tree. Only PCNA-type fruit loses astringency regardless of the presence or absence of seeds (Kitagawa and Glucina, 1984). Furthermore, the quality of PVNAtype fruit usually is not as good as that of PCNA-type fruit. Hence, PCNA-type fruit is the most desirable for the persimmon industry and the demand for new PCNA-type cultivars having good fruit

Received for publication 20 Sept. 1999. Accepted for publication 6 Sept. 2000 This research was partially supported by a grant-in-aid (no. 09556008) for scientific research (B) from the Ministry of Education, Science, Sports and Culture, Japan. The cost of publishing this paper was defrayed in part by the payment of page charges. Under postal regulations, this paper therefore must be hereby marked advertisement solely to indicate this fact.

${ }^{1}$ Current address: Laboratory of Horticulture, Faculty of Agriculture, Kinki University, Nakamachi, Nara 631-8505, Japan.

${ }^{2}$ Corresponding author; e-mail: keizo@kais.kyoto-u.ac.jp. quality is increasing worldwide.

The PCNA type is qualitatively different from the other three types in the level of tannin accumulation in the fruit. Tannin is accumulated in the vacuole of tannin cells, which are idioblasts of parenchyma cells in the flesh. The PCNA-type fruit accumulates less tannin and the tannin cells are much smaller than in the other three types (Yonemori and Matsushima, 1985). PCNA-type fruit stops tannin accumulation at a very early stage of the development, whereas fruit of the other three types continue to accumulate tannin until the middle stage of development. The natural astringency loss in PCNA-type fruit is caused mainly by dilution of tannin in the fruit due to the early cessation of tannin accumulation, although tannin coagulation in PCNA-type fruit also occurs at maturity by further oxidative reactions (Yonemori and Matsushima, 1985). The low tannin accumulation is responsible for the trait of natural astringency loss in PCNA-type fruit.

The PCNA genotype appears to be homozygous recessive for the natural loss of astringency, since the trait of natural astringency-loss in PCNA-type fruit is qualitatively inherited to the progenies and the PCNA genotype is recessive to the other three types (Ikeda et al., 1985). Even in the progenies from crosses between PVNA-type and PCNA-type cultivars, no PCNA-type offspring were obtained, although the other three types yielded variable ratios (Ikeda et al., 1985). So far, PCNA-type cultivars/ selections have been intercrossed to obtain exclusively PCNAtype offspring in $\mathrm{F}_{1}$ progenies in the breeding program in Japan. However, it has been difficult to recover excellent PCNA-type offspring by intercrossing PCNA-type plants. In addition, the genetic base of the existing PCNA-type cultivars is very narrow because of their recent origin (Yamada, 1993), resulting in inbreeding depression in such crosses (Yamada et al., 1994). Some PCNA-type cultivars producing male or hermaphrodite flowers also limit parent combinations among PCNA genotypes. 


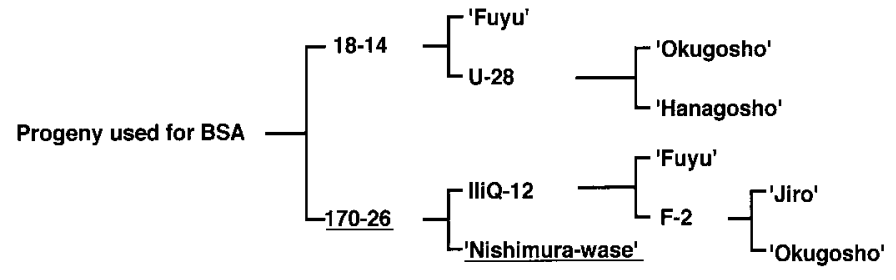

Fig. 1. Pedigree of the progeny used for bulked segregant analysis (BSA). The non-PCNA-type cultivar/selection is underlined. The other are PCNA-type cultivars/selections.

On the other hand, non-PCNA-type cultivars have wide genetic variability with some attractive characters that PCNA-type cultivars do not have. To widen the genetic base of PCNA-type cultivars and introduce the improved characters of non-PCNA type cultivars, we should utilize non-PCNA type plants in PCNA breeding. However, among the $F_{1}$ progeny from the crosses between some existing PCNA-type and non-PCNA-type cultivars, none were of the PCNAtype (Ikeda et al., 1985). When non-PCNA-type $\mathrm{F}_{1}$ plants were crossed with a PCNA-type clone, few PCNA-type plants (15\%) were found in the progeny (Ikeda et al., 1985), as shown in recent breeding programs at the Persimmon and Grape Research Station, Akitsu, Japan (unpublished data). This low percentage of obtainable PCNA-type offspring indicates that some loci are involved with the PCNA/non-PCNA trait. Two or three genes may be associated with this trait. In addition, Japanese persimmon is a hexaploid (Namikawa and Higashi, 1928; Zhuang et al., 1990) and, so far, there is no consensus whether it is an auto-, allo- or autoallo-hexaploid and whether the mode of inheritance is disomic or polysomic. Based on observations of chromosome behavior of pollen mother cells at meiosis, Zhuang (1990) hypothesized that Japanese persimmon is not an autohexaploid. She reported that bivalent formation occurred regularly, and few multivalents and secondary associations of bivalent were observed at meiosis. However, lack of multivalents does not necessarily indicate a disomic polyploid (Krebs and Hancock, 1989; Qu et al., 1998; Wolf et al., 1989). The low percentage of PCNA-type offspring in the progeny from the cross between PCNA-type and non-PCNA-type $F_{1}$ plants makes selection of PCNA-type plants a laborious task, since the phenotype can be evaluated only after the tree has entered fruiting age.

Marker-assisted selection is considered a useful tool in plant breeding, especially in tree crops (Bernatzky and Mulcahy, 1992). Recently, molecular markers linked to some important traits of tree fruits have been identified, such as markers associated with apple scab [Venturia inaequalis (Cke.) Wint.] resistance (Gardiner et al., 1996; Yang et al., 1997), seedlessness in grape (Vitis vinifera L.) (Striemetal., 1996), resistance to root-knotnematodes (Meloidogyne sp.) in peach [Prunus persica (L.) Batsch] rootstocks (Lu et al., 1998), and ethylene levels in ripening Japanese pear (Pyrus pyrifolia Nakai) fruit (Itai et al., 1999). We are aiming to develop a markerassisted selection system for persimmon breeding. In this study, we identified molecular markers specific for the non-PCNA trait by using amplified fragment length polymorphism (AFLP) techniques (Vos et al., 1995). Furthermore, the possibility of polysomic inheritance of Japanese persimmon is discussed based on segregation of the two markers linked to each of the two dominant alleles.

\section{Materials and Methods}

Plant materials. All plant materials were from the breeding programs at the Persimmon and Grape Research Center, National
Institute of Fruit Tree Science, Akitsu, Japan. The progeny population used in this experiment was derived from the cross between two selections, 18-14 [PCNA-type $\mathrm{F}_{1}$ from crossing 'Fuyu' (PCNA-type cultivar) and U-28 (PCNA-type clone)] and 170-26 [non-PCNA-type $F_{1}$ from crossing IIiQ-12 (PCNA-type) and 'Nishimura-wase' (non-PCNA-type cultivar)] (Fig.1). As PCNA and non-PCNA genotypes segregated in this population, bulked segregant analysis (BSA) (Michelmore et al., 1991) was carried out by pooling DNA of six PCNA-type plants as a PCNA bulk and of 10 non-PCNA plants as a non-PCNA bulk. PCNAtype and non-PCNA-type plants from other backcross populations (donor parent: 'Nishimura-wase', recurrent parents: some PCNA-type clones) were also used for evaluating the segregation of markers.

DNA EXTRACTION AND AFLP ANALYSIS. Total DNAs were extracted from leaves of each plant by the CTAB method according to Doyle and Doyle (1987). After purifying DNAs by phenol extraction and polyethylene glycol (PEG) precipitation, DNA concentration was adjusted to $50 \mathrm{ng} \cdot \mu \mathrm{L}^{-1}$ for AFLP analysis.

DNA digestion, adapter ligation, and preamplification were performed as described by Vos et al. (1995) using the AFLP analysis system I kit (Life Technologies, Gaithersburg, Md.). Total genomic DNA $(0.25 \mu \mathrm{g})$ of each bulked sample, each parent, and each individual offspring was digested with 2.5 unit EcoRI and 2.5 unit $M s e \mathrm{I}$ in $25 \mu \mathrm{L}$ reaction mixtures. Then, EcoRI and $M s e I$ adapters were ligated to the digested DNA fragments. After the preamplification reaction with $5 \mu \mathrm{L}$ of template DNA (1:10, v/v solution diluted from the restriction-ligation mixture), the selective amplification reaction was performed with two primers based on the sequence of EcoRI and MseI adapters including three additional selective nucleotides at the 3' end of each primer. Eight EcoRI and MseI primers in the kit and additional eight $M s e I$ primers were used for screening with the two bulks (Table 1).

After selective amplifications, the PCR products were denatured by boiling with an equal volume of tracking dye [98\% formamide, $10 \mathrm{~mm}$ ethylenediaminetetraacetic acid (EDTA), $0.05 \%$ bromophenol blue, and $0.05 \%$ xylene cyanol]. Then, aliquots $(4 \mu \mathrm{L})$ of each reaction were loaded onto denaturing $6 \%$ polyacrylamide gel (acrylamide-bisacrylamide 29:1). The gel was run at a constant voltage $\left(200 \mathrm{~V}, 10 \mathrm{~V} \cdot \mathrm{cm}^{-1}\right)$. Thereafter, the amplified fragments were transferred to a Biodyne B membrane (Pall, Inc., Port Washington, N.Y.). The membrane was then hybridized with an AFLP nonradioactive probe (Life Technologies), which hybridizes to the EcoRI adapter sequences, according to the manufacturer's manual. After being washed, the membrane was incubated with CDP-Star (Boehringer Mannheim, Indianapolis, Ind.) at $37^{\circ} \mathrm{C}$ for $15 \mathrm{~min}$ and exposed to X-ray film for 3 to $7 \mathrm{~h}$.

Cloning ANd Sequencing of The AFlP MARKer. The most

Table 1. Eight Eco primers and 16 Mse primers used for BSA.

\begin{tabular}{lll}
\hline \hline Eco primer & Mse primer & \\
\hline EAAC & MCAA & MCGA \\
EAAG & MCAC & MCGT \\
EACA & MCAG & MCGG \\
EACT & MCAT & MCGC \\
EACC & MCTA & MCCA \\
EACG & MCTC & MCCT \\
EAGC & MCTG & MCCG \\
EAGG & MCTT & MCCC \\
\hline
\end{tabular}




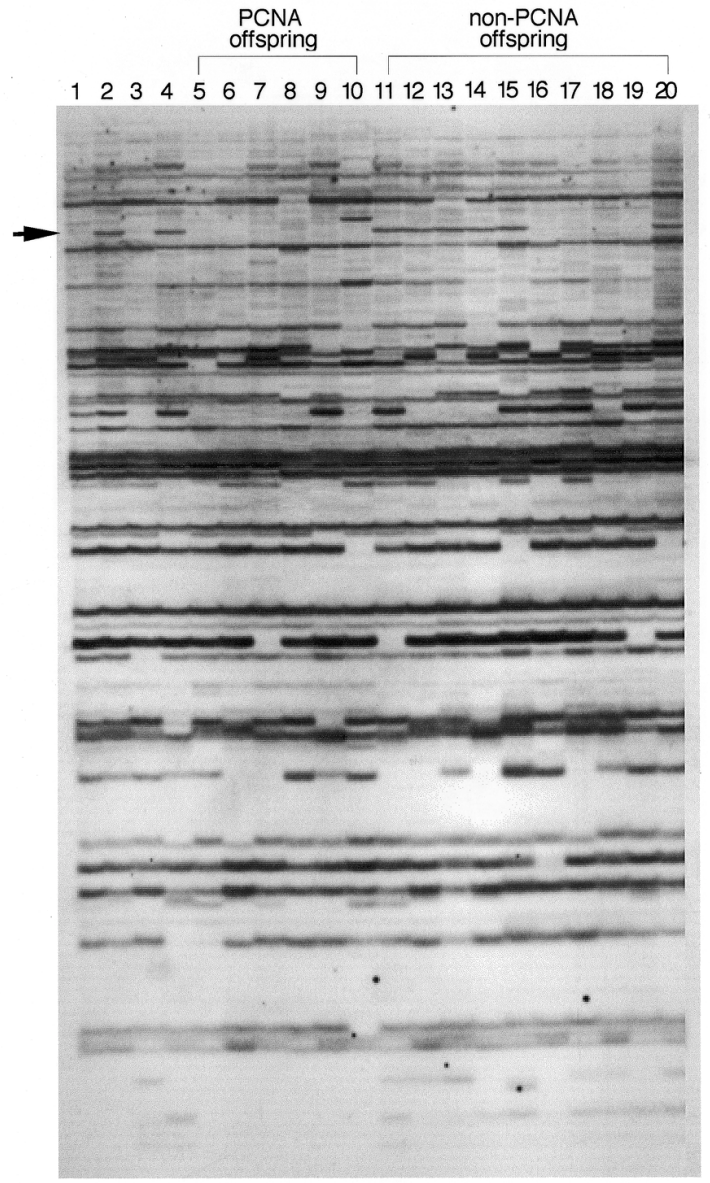

Fig. 2. AFLP fingerprints of PCNA and non-PCNA bulks, parents, and individual offspring for bulked segregant analysis (BSA), using primer combination EACC/MCTA: Lanes: 1, PCNA bulk; 2, non-PCNA bulk; 3, 18-14 (PCNAtype parent); 4, 170-26 (non-PCNA-type parent); 5-10, PCNA-type offspring; 11-20, non-PCNA-type offspring. Arrow indicates the AFLP marker, EACC/ MCTA-400.

reliable AFLP marker in the present experiment was recovered from the gel. For this purpose, the gel was stained with SYBR Gold Nucleic Acid Gel Stain (Molecular Probes, Eugene, Ore.) after electrophoresis, and the marker band was excised under ultraviolet light. After the addition of $20 \mu \mathrm{L}$ sterilized-distilled water, the gel piece was incubated on ice for $10 \mathrm{~min}$ and then at $94^{\circ} \mathrm{C}$ for $20 \mathrm{~min}$. After a brief centrifugation, $2 \mu \mathrm{L}$ of the resulting supernatant was reamplified with the same primer combination, EACC/MCTA. The reamplified fragment was subcloned into pGEM-T easy vector (Promega, Madison, Wis.) and the recombinant clones were screened for the appropriate size of insert before sequencing. Both ends of each DNA insert were sequenced using an automatic DNA sequencer (model 373A; Applied Biosystems, Perkin Elmer, Foster City, Calif.).

SOUTHERN BLOT ANALYSIS. Based on the nucleotide sequences of the most reliable AFLP marker, a pair of primers was designed and PCR was performed by using the AFLP fragment cloned into pGEM-T vector as a template. The PCR products were DIGdUTP-labeled with these primers using a PCR DIG Probe Synthesis Kit (Boehringer Mannheim) and were used as a probe for Southern analysis. For Southern analysis, $20 \mu \mathrm{g}$ of total genomic DNAs were digested with an appropriate restriction enzyme and run on $0.8 \%$ agarose gel, transferred to a nylon membrane (Hybond-N; Amersham, Buckinghamshire, United Kingdom), and hybridized with the DIG-labeled probe at $65^{\circ} \mathrm{C}$ for $16 \mathrm{~h}$. After high stringency washes $\left[2 \times 5\right.$ min at $25^{\circ} \mathrm{C}$ with $2 \times$ saline sodium citrate (SSC) and $0.1 \%$ sodium dodecyl sulfate (SDS) followed by $2 \times 15 \mathrm{~min}$ at $65{ }^{\circ} \mathrm{C}$ with $0.1 \times \mathrm{SSC}$ and $0.1 \% \mathrm{SDS}$ ], the hybridization was analyzed immunologically using the antiDIG-alkaline phosphatase conjugate and the chemiluminescent substrate CDP-Star (Boehringer Mannheim).

\section{Results and Discussion}

AfLP SCREENING. A total of 128 primer combinations of eight EcoRI and $16 \mathrm{MseI}$ primers were used in a bulked segregant analysis (BSA) against subsets of PCNA and non-PCNA bulks. About 40 bands were detected per primer combination and 56 polymorphic markers were identified between the two bulk populations (absent in PCNA bulk and present in non-PCNA bulk). After these 56 candidate markers were examined against the individual parents and offspring used for BSA, four primer combinations (EACC/MCTA, EAAC/MCCC, EAAG/MCGG, and EACA/MCGA) proved to generate four potential AFLP markers which were absent in 18-14 (PCNA-type parent) and all individuals from the PCNA bulk and present in 170-26 (nonPCNA-type parent) and some individuals from the non-PCNA bulk. The AFLP marker generated by EACA/MCGA was present in only three of the 10 non-PCNA-type offspring, and the markers generated by the other three primer combinations were present in six of the 10 non-PCNA-type offspring (Fig. 2).

Within the 35 additional progeny (20 PCNA-type and 15 non-

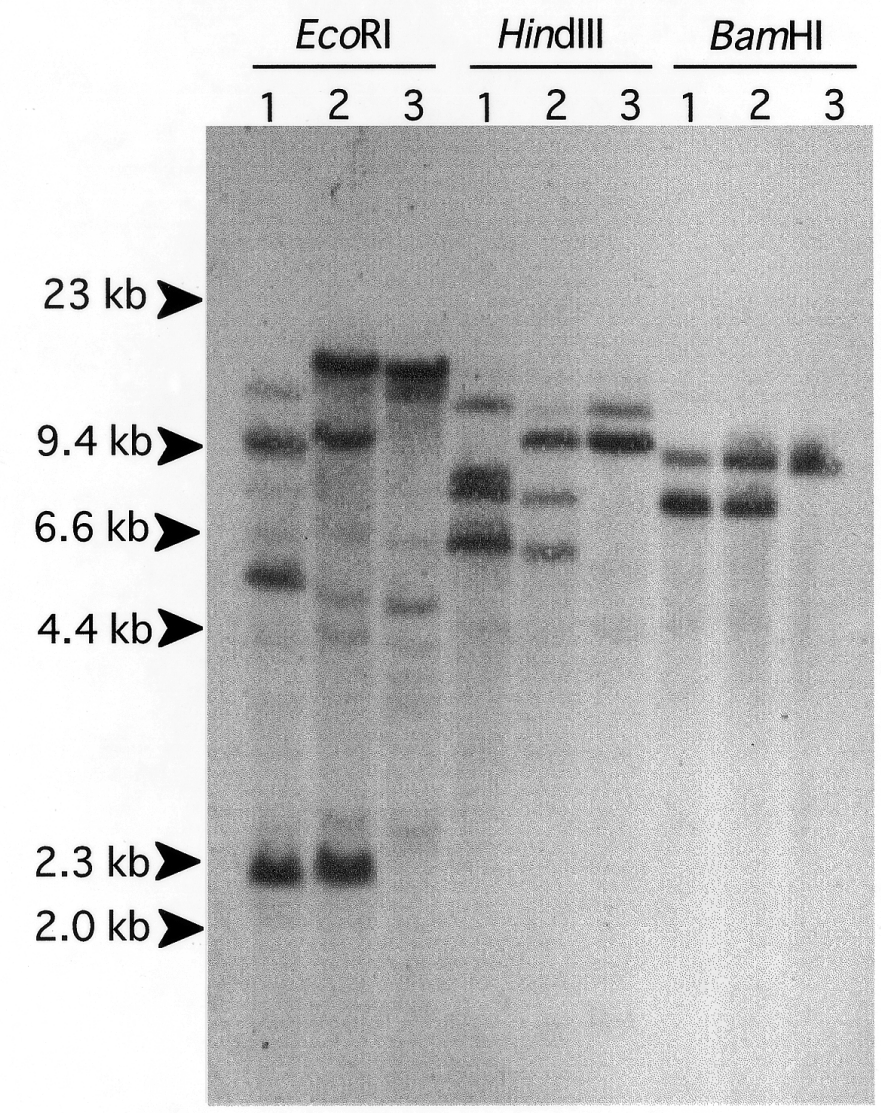

Fig. 3. RFLP analysis of genomic DNAs digested with EcoRI, HindIII and BamHI, using EACC/MCTA-400 as a probe. Lanes: 1, 'Nishimura-wase'; 2, a non-PCNA-type offspring; 3, a PCNA-type offspring. 


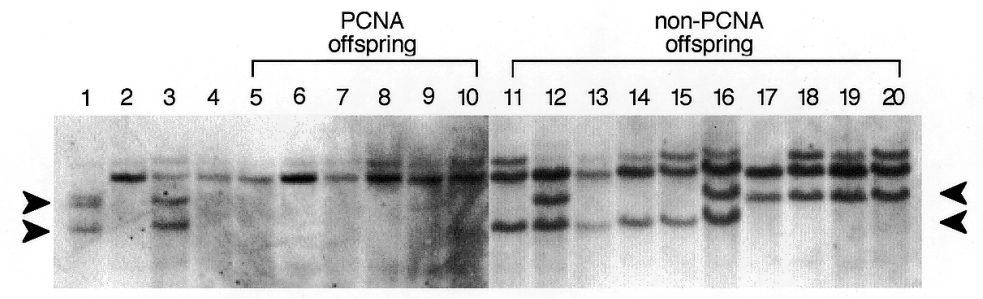

Fig. 4. RFLP analysis of genomic DNAs digested with HindIII, using EACC/ MCTA-400 as a probe. Lanes: 1, 'Nishimura-wase'; 2, IIiQ-12; 3, 170-26; 4, 18-14; 5-10, PCNA-type offspring used for BSA; 11-20, non-PCNA-type offspring used for BSA. Arrows indicate the $8 \mathrm{~kb}$ (upper) and $6.5 \mathrm{~kb}$ (lower) RFLP markers.

PCNA-type) derived from four other populations, the markers from two primer combinations (EAAG/MCGG and EAAC/ $\mathrm{MCCC}$ ) did not cosegregate with PCNA/non-PCNA phenotypes. These markers were eliminated in further analysis. The marker generated by EACA/MCGA was absent in all 20 PCNA-type individuals tested and present in four of the 15 non-PCNA-type individuals. Including 10 non-PCNA-type offspring used in BSA, this marker was present in seven of the 25 non-PCNA-type offspring. The marker generated by EACC/MCTA, with the size of $\approx 400$ base pairs (bp), was present in seven of the 15 nonPCNA-type offspring tested and also absent in all PCNA-type offspring. This marker was present in 13 of the 25 non-PCNAtype offspring tested and absent in all 26 PCNA-type plants. As all PCNA-type plants tested were negative and half of the nonPCNA-type plants were positive to the AFLP marker (EACC/ MCTA-400), we investigated further whether it is indeed associated to the non-PCNA trait.

SOUTHERN BLOT ANALYSIS. The most reliable AFLP marker (EACC/MCTA-400) was recovered from the polyacrylamide gel and subcloned into the pGEM-T easy vector. Identity of the cloned products was verified by checking the size of the PCR product using the EACC/MCTA primer combination. The sequencing result showed that EACC/MCTA-400 had a size of 403 bp between the EcoRI and $M s e$ I sites. Based on sequencing, a pair of sequence-specific primers was designed. When these primers were used for PCR with the individual DNAs of both PCNA-type and non-PCNA-type offspring used for BSA, an identical single fragment with the size expected was amplified in all individuals tested (data not presented). This indicates that a few mutations had occurred on the restriction sites flanking EACC/MCTA-400, or adjacent regions of these restriction sites. These mutations could be detected by AFLP analysis, but not by PCR with the specific primer set. Thus, PCR analysis using these sequencespecific primers could not distinguish PCNA-type plants from non-PCNA-type plants in the breeding population. However, RFLP analysis of the EcoRI, HindIII, and BamHI digests from 'Nishimura-wase', a PCNA-type offspring, and a non-PCNAtype offspring using EACC/MCTA-400 as a probe revealed polymorphic bands between PCNA and nonPCNA plants (Fig. 3). One to four bands were detected with each restriction enzyme digest. Some of the bands were present only in 'Nishimura-wase' and a non-PCNA plant.

After digestion with HindIII, all 10 nonPCNA-type offspring used for BSA and the non-PCNA-type parent (170-26) showed the band(s) of 6.5 or $8 \mathrm{~kb}$, or both, (Fig. 4), whereas the six PCNA-type offspring for
BSA and the PCNA-type parent (18-14) showed neither of the bands (Fig. 4). The segregation pattern of the $6.5 \mathrm{~kb}$ band was the same as that of EACC/MCTA-400 obtained by AFLP analysis. The $8 \mathrm{~kb}$ band could be detected in six non-PCNA-type offspring, four of which did not have the EACC/MCTA-400 marker in the AFLP analysis. These results indicate that the $6.5 \mathrm{~kb}$ RFLP marker is linked to the same dominant allele as the AFLP marker, EACC/MCTA400 , and that the other $8 \mathrm{~kb}$ RFLP marker is linked to another dominant allele.

In the RFLP analysis of the progenies from other populations, HindIII digests of 13 PCNA-type offspring revealed no polymorphism and neither the 6.5 nor $8 \mathrm{~kb}$ band was detected (Fig. 5), as in the banding pattern of the six PCNA-type offspring used for BSA. On the other hand, the non-PCNA-type plants showed polymorphic bands and had either a 6.5 or $8 \mathrm{~kb}$ band, or both (Fig. 5 ), as in the 10 non-PCNA-type offspring used for BSA. Thus, we could distinguish the PCNA-type plants from the non-PCNAtype plants in all 42 individuals tested with $100 \%$ consistently.

The trait of natural astringency loss is controlled by some loci as suggested from the low percentage of obtainable PCNA-type offspring in populations from the cross between PCNA-type and non-PCNA-type $F_{1}$ plants. Ikeda et al. (1985) reported $15 \%$ PCNA-type plants were recovered when non-PCNA-type $F_{1}$ plants derived from the PCNA-type and non-PCNA-type cultivars were backcrossed to PCNA-type cultivars. Similarly, $18 \%$ was reported in another such cross (PCNA-type with non-PCNAtype $\mathrm{F}_{1}$ from 'Nishimura-wase' $\times$ PCNA clone) in recent breeding programs at the Persimmon and Grape Research Station, Akitsu (unpublished data). In the present study, we could distinguish the PCNA-type offspring from the non-PCNA-type offspring by the presence or absence of two RFLP markers, which appeared to link to a different dominant allele. This suggests that there are at least two DNA fragments which are associated separately with gene(s) controlling the non-PCNA phenotype, and that the gene linked with each fragment is able to express the trait. It is clear that the two DNA fragments are not located on the same chromosome, since, if this were the case, $50 \%$ to $70 \%$ of the backcross progeny would be non-PCNA-type plants, depending on the genetic distance between them. In addition, if Japanese persimmon is considered as an allohexaploid and the donor parent (non-PCNA type) was a homozygous dominant genotype with duplicate genes conferring this phenotypic trait, the expected segregation of the non-PCNA type to PCNA type would be 3 to 1 . However, the results of a previous study (Ikeda et al., 1985) and recent breeding programs in Akitsu (unpublished data) deviate from this expected ratio. Our results indicate that Japanese persimmon has more than

Fig. 5. RFLP analysis of PCNA-type and non-PCNA-type offspring derived from different parent combinations with HindIII digests. Lanes: 1-13, PCNA-type offspring; 14-26, non-PCNA-type offspring. Arrows indicate $8 \mathrm{~kb}$ (upper) and $6.5 \mathrm{~kb}$ (lower) RFLP markers. 
two homologous chromosomes in a homologous group, since two chromosomes, each carrying one of the fragments, are inherited from one parent (non-PCNA) and two DNA fragments seemed to segregate nonindependently in the progeny. If Japanese persimmon is an auto- or autoallo-hexaploid and two alleic genes are present in the non-PCNA-type $\mathrm{F}_{1}$, polysomic inheritance is possible and the ratio would fit the assumption. For example, if Japanese persimmon is considered dibasic hexaploid and the nonPCNA-type $F_{1}$ has two dominant alleles among four at a locus controlling the PCNA/non-PCNA trait, $16.7 \%$ to $21.4 \%$ of the backcross progeny would be expected to be PCNA-type plants. Our findings are not sufficient to determine whether Japanese persimmon is an autohexaploid or autoallohexaploid, but strongly imply that Japanese persimmon is not an allohexaploid.

In conclusion, production of new and commercially superior PCNA-type Japanese persimmon cultivars is the most important objective for persimmon breeding. Although the necessity of using non-PCNA types in PCNA breeding programs has been recognized, it is difficult to achieve progress in such programs without an efficient system for selecting PCNA-type plants. Only a small number of PCNA-type plants can be obtained even in the backcross populations. Furthermore, the conventional selection system is laborious and time-consuming. We believe that our finding of molecular markers linked to the trait of natural astringency loss will be very helpful in persimmon breeding by permitting efficient selection of PCNA-type plants.

\section{Literature Cited}

Bernatzky, R. and D.L. Mulcahy. 1992. Marker-aided selection in a backcross breeding program for resistance to chestnut blight in the American chestnut. Can. J. For. Res. 22:1031-1035.

Doyle, J.J. and J.L. Doyle. 1987. A rapid DNA isolation procedure for small quantities of fresh leaf tissue. Phytochem Bul. 19:11-15.

Gardiner, S.E., H.C.M. Bassett, D.A.M. Noiton, V.G. Bus, M.E. Hofstee, and A.G. White. 1996. A detailed linkage map around an apple scab resistance gene demonstrates that two disease resistance classes both carry the $V f$ gene. Theor. Appl. Genet. 93:485-493.

Ikeda, I., M. Yamada, A. Kurihara, and T. Nishida. 1985. Inheritance of astringency in Japanese persimmon (in Japanese with English summary). J. Jpn. Soc. Hort. Sci. 54:39-45.

Itai, A., T. Kawata, K. Tanabe, F. Tamura, M. Uchiyama, M. Tomomitsu, and N. Shiraiwa. 1999. Identification of 1-aminocyclopropane-1carboxylic acid synthase genes controlling the ethylene level of ripening fruit in Japanese pear. Mol. Gen. Genet. 261:42-49.

Kajiura, M. 1946. Persimmon cultivars and their improvement (2) (in Japanese). Breeding Hort. 1:175-182.
Kikuchi, A. 1948. Pomology_Part I (in Japanese). Yokendo, Tokyo, Japan.

Kitagawa, H. and P.G. Glucina. 1984. Persimmon culture in New Zealand. Sci. Info. Publ. Ctr., DSIR, Wellington, N.Z.

Krebs, S.L. and J.F. Hancock. 1989. Tetrasomic inheritance of isoenzyme markers in the highbush blueberry, Vaccinium corymbosum $\mathrm{L}$. Heredity 63:11-18.

Lu, Z.X., B. Sosinski, G.L. Reighard, W.V. Baird, and A.G. Abbott. 1998. Construction of a genetic linkage map and identification of AFLP markers for resistance to root-knot nematodes in peach rootstocks. Genome 41:199-207.

Michelmore, R.W., I. Paran, and R.V. Kesseli. 1991. Identification of markers linked to disease-resistance genes by bulked segregant analysis: A rapid method to detect markers in specific genomic regions by using segregation populations. Proc. Natl. Acad. Sci. USA 88:98289832.

Namikawa, I. and M. Higashi. 1928. On the number of chromosomes in Diospyros kaki L.f. and Diospyros lotus L. Bot. Mag. 42: 436-438.

Qu, L., J.F. Hancock, and J.H. Whallon. 1998. Evolution in an autopolyploid group displaying predominantly bivalent pairing at meiosis: Genomic similarity of diploid Vaccinium darrowi and autotetraploid V. corymbosum (Ericaceae). Amer. J. Bot. 85: 698-703.

Striem, M.J., H.G. Ben, and P. Spiegel-Roy. 1996. Identifying molecular genetic markers associated with seedlessness in grape. J. Amer. Soc. Hort. Sci. 121:758-763.

Vos, P., R. Hogers, M. Bleeker, M. Reijans, T. Lee, M. Hornes, A. Frijters, J. Pot, J. Peleman, M. Kuiper, and M. Zabeau. 1995. AFLP: A new technique for DNA fingerprinting. Nucleic Acids Res. 23:44074414.

Wolf, P.G.,P.S. Soltis, and D.E. Soltis. 1989. Tetrasomic inheritance and chromosome pairing behavior in the naturally occurring autotetraploid Heuchera grossulariifolia (Saxifragaceae). Genome 32:655-659.

Yamada, M. 1993. Persimmon breeding in Japan. Jpn. Agr. Res. Quarterly 27:33-37.

Yamada, M., H. Yamane, and Y. Ukai. 1994. Genetic analysis of Japanese persimmon fruit weight. J. Amer. Soc. Hort. Sci. 119:12981302.

Yang, H.Y., S.S. Korban, J. Krueger, and H. Schmidt. 1997. A randomly amplified polymorphic DNA (RAPD) marker tightly linked to the scab-resistance gene $V f$ in apple. J. Amer. Soc. Hort. Sci. 122:47-52. Yonemori, K. and J. Matsushima. 1985. Property of development of the tannin cells in nonastringent type fruits of Japanese persimmon (Diospyros kaki) and its relationship to natural deastringency (in Japanese with English summary). J. Jpn. Soc. Hort. Sci. 54:201-208.

Zhuang, D. H. 1990. Cytogenetic studies on Japanese persimmon cultivars-On the chromosome number of seedless cultivars (in Japanese with English summary). PhD diss., Kyoto Pref. Univ., Kyoto, Japan.

Zhuang, D. H., A. Kitajima, M. Ishida, and Y. Sobajima. 1990. Chromosome numbers of Diospyros kaki cultivars (in Japanese with English summary). J. Jpn. Soc. Hort. Sci. 59:289-297. 